\title{
Near-Field Correlation Measurement and Evaluation of Stationary and Cyclostationary Stochastic Electromagnetic Fields
}

\author{
Johannes A. Russer*1, Michael Haider*, Mohd H. Baharuddin ${ }^{\dagger}$, Christopher Smartt ${ }^{\dagger}$, Sidina Wane ${ }^{\ddagger}$, \\ Damienne Bajon ${ }^{\S}$, Andrey Baev $₫$, Yury Kuznetsov $₫$, David Thomas ${ }^{\dagger}$, and Peter Russer* \\ ${ }^{*}$ Institute for Nanoelectronics, Technische Universität München, Germany \\ ${ }^{\dagger}$ University of Nottingham, School of Electrical and Electronic Engineering, Nottingham \\ ${ }^{\ddagger}$ NXP-Semiconductors, Caen-France \\ $\S$ ISAE-Universite de Toulouse, Toulouse-France \\ ITheoretical Radio Engineering Department, Moscow Aviation Institute, Moscow, Russia \\ 1 jrusser@tum.de
}

\begin{abstract}
We present methods for measurement and evaluation of stationary and cyclostationary stochastic electromagnetic fields. The radiated electromagnetic interference (EMI) of electronic circuitry is recorded by two-point measurements of the tangential electric or magnetic field components and by evaluating the field autocorrelation functions and for each pair of field sampling points also the cross correlation functions. In case of digital circuitry clocked by a single clock pulse, the generated EMI is a cyclostationary process where the expectation values of the EMI are periodically time dependent according to the clock frequency and which have to be considered in modeling the EMI.
\end{abstract}

\section{INTRODUCTION}

Electromagnetic interference (EMI) radiated by electronic circuitry due to switching operations of the active circuit elements degrades the performance of electronic circuits and systems. Modeling the EMI radiated by integrated circuits or circuit boards into a system to be designed, requires the consideration of the stochastic EM field, usually on the basis of the measured radiated EMI of the components, and to establish models based on these measurements. Such models already were discussed in literature [1]-[4]. Sampling of stochastic EM fields and source localization have been addressed in [4][7]. Numerical simulation methodologies based on the field auto- and cross correlation spectra were developed [8]-[13].

In digital circuitry switching operations synchronized by a periodic clock generate an EMI described by a cyclostationary (CS) process i.e. a process where the expectation values of the EMI are periodically time dependent on the clock frequency [14]-[18]. This has to be considered in modeling the influence of the EMI. Figure 1 shows an example of a CS random signal and of the time dependence of its variance.

Due to the equivalence principle, an equivalent source distribution determined by amplitude and phase scanning of the tangential electric or magnetic field on a surface enclosing the radiating structure is equivalent to the internal sources and allows the modeling of the environmental field. However, due

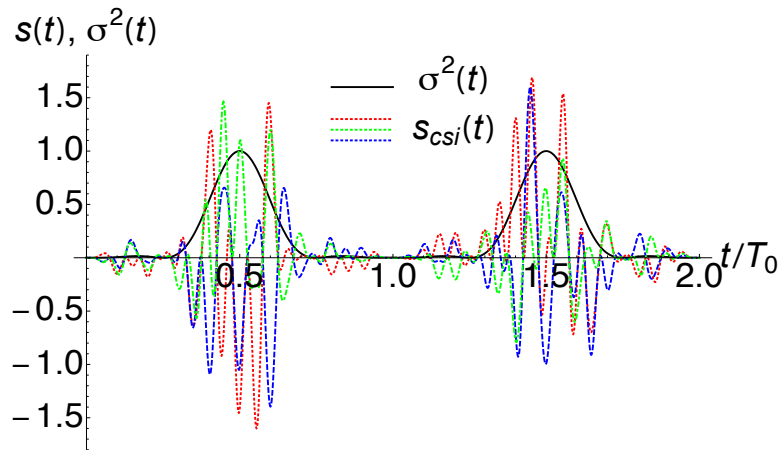

Fig. 1: Time of three samples of a CS random signal $s_{c s 1}$ with period $T_{0}$ and of the time dependence of its variance $\sigma^{2}(t)$.

to the lack of information about the sources of the radiated EMI, the near field to be measured has to be treated as a stochastic field. The radiated EMI of electronic circuitry is recorded by two-point measurements of the tangential electric or magnetic field components and by evaluating the field autocorrelation functions and for each pair of field sampling points also the cross correlation functions. In [19], [20], we have extended the near-field correlation measurement methods to CS EM fields. In this work we present experimental investigations of CS noise radiated by electric and electronic components, namely due to the data transfer process of a microcontroller board, and the recording and evaluation of CS radiated EMI using two-point scanning methods.

\section{The CyClOSTATIONARY EM FIELD}

The time-domain correlation dyadic of two electric field variables $\tilde{\boldsymbol{E}}\left(\boldsymbol{x}_{1}, t_{1}\right)$ and $\tilde{\boldsymbol{E}}\left(\boldsymbol{x}_{2}, t_{2}\right)$ at points $\boldsymbol{x}_{a}$ and $\boldsymbol{x}_{b}$, at times $t_{1}$ and $t_{2}$, respectively is given by

$$
\tilde{\underline{\Gamma}}_{E}\left(\boldsymbol{x}_{a}, \boldsymbol{x}_{b}, t_{1}, t_{2}\right)=\lim _{T \rightarrow \infty} \frac{1}{2 T}\left\langle\tilde{\boldsymbol{E}}_{T}\left(\boldsymbol{x}_{a}, t_{1}\right) \tilde{\boldsymbol{E}}_{T}^{\dagger}\left(\boldsymbol{x}_{b}, t_{2}\right)\right\rangle,
$$

where the subscript $T$ denotes the amplitude spectrum of the field time-windowed by a rectangular window covering 
the time interval $[-T, T]$. The tilde $\sim$ denotes time-domain representation whereas field variables without tilde refer to frequency domain representation.

If the mean value of the field $\tilde{\boldsymbol{E}}(\boldsymbol{x}, t)$ and also the correlation dyadic describing the second-order statistics are periodic with a period $T_{0}$, i.e.

$$
\begin{aligned}
\left\langle\boldsymbol{E}\left(\boldsymbol{x}, t+T_{0}\right)\right\rangle & =\langle\boldsymbol{E}(\boldsymbol{x}, t)\rangle, \\
\underline{\underline{\Gamma}}_{E}\left(\boldsymbol{x}_{a}, \boldsymbol{x}_{b}, t_{1}+T_{0}, t_{2}+T_{0}\right) & =\underline{\underline{\Gamma}}_{E}\left(\boldsymbol{x}_{a}, \boldsymbol{x}_{b}, t_{1}, t_{2}\right),
\end{aligned}
$$

the field is said to be second-order cyclostationary in the wide sense [14]-[18].

Applying discrete-time measurement systems yields a discretization of the measurement quantities. A discrete-time second-order random process $X[n] \forall n \in \mathbb{N}$ is defined to be cyclostationary with period $N$ if and only if its mean and autocorrelation are periodic [15], [21], [22]:

$$
\begin{aligned}
\mu_{X}[n] & \equiv\langle X[n]\rangle=\mu_{X}[n+k N] \quad \forall k \in \mathbb{N}, \\
\rho_{X}[n, m] & \equiv\left\langle X[n] X^{*}[n-m]\right\rangle \\
& =\rho_{X}[n+k N, m] \quad \forall n, m \in \mathbb{N} .
\end{aligned}
$$

\section{Spectral Representation of the CS EM Field}

Expanding the correlation dyadic, periodic in time with $T_{0}$, into a Fourier series yields

$$
\underline{\underline{\Gamma}}_{E}\left(\boldsymbol{x}_{a}, \boldsymbol{x}_{b}, t, t-\tau\right)=\sum_{n=-\infty}^{+\infty} \underline{\underline{\Gamma}}_{E, n}\left(\boldsymbol{x}_{a}, \boldsymbol{x}_{b}, \tau\right) \mathrm{e}^{\jmath n \omega_{0} t},
$$

with $\omega_{0}=2 \pi / T_{0}$. The Fourier coefficients

$$
\underline{\underline{\Gamma}}_{E, n}\left(\boldsymbol{x}_{a}, \boldsymbol{x}_{b}, \tau\right)=\frac{1}{T_{0}} \int_{-T_{0} / 2}^{T_{0} / 2} \underline{\underline{\Gamma}}_{E}\left(\boldsymbol{x}_{a}, \boldsymbol{x}_{b}, t, t-\tau\right) \mathrm{e}^{-\jmath n \omega_{0} t} d t
$$

are referred to as the cyclic time-domain autocorrelation dyadics and the frequencies $n f_{0}=n \omega_{0} / 2 \pi$ are the so-called cycle frequencies [15], [19]. The cyclic correlation spectrum (CCS) of the electric field $\underline{\underline{\Gamma}}_{E, n}\left(\boldsymbol{x}_{a}, \boldsymbol{x}_{b}, \omega\right)$ is given by

$$
\underline{\underline{\Gamma}}_{E, n}\left(\boldsymbol{x}_{a}, \boldsymbol{x}_{b}, \omega\right)=\int \underline{\underline{\Gamma}}_{E, n}\left(\boldsymbol{x}_{a}, \boldsymbol{x}_{b}, \tau\right) \mathrm{e}^{-\jmath \omega \tau} d \omega .
$$

The inverse Fourier transform yields the cyclic time-domain correlation dyadic

$$
\underline{\underline{\Gamma}}_{E, n}\left(\boldsymbol{x}_{a}, \boldsymbol{x}_{b}, \tau\right)=\int \underline{\underline{\Gamma}}_{E, n}\left(\boldsymbol{x}_{a}, \boldsymbol{x}_{b}, \omega\right) \mathrm{e}^{\jmath \omega \tau} d \omega .
$$

\section{EXCITATION OF A CS EM FIELD}

Consider point-like CS stochastic EMI sources $S_{i}$ arranged as shown in Fig. 2. Describing a deterministic source current distribution by the source current density $\boldsymbol{J}(\boldsymbol{x}, \omega)$, the excited electric field $\boldsymbol{E}(\boldsymbol{x}, \omega)$ is given by

$$
\boldsymbol{E}(\boldsymbol{x}, \omega)=\int_{V} \boldsymbol{G}_{E J}\left(\boldsymbol{x}-\boldsymbol{x}^{\prime}, \omega\right) \boldsymbol{J}\left(\boldsymbol{x}^{\prime}, \omega\right) d^{3} x^{\prime},
$$

where $\boldsymbol{G}_{E J}\left(\boldsymbol{x}-\boldsymbol{x}^{\prime}, \omega\right)$ is the Green's dyadic relating the excited electric field $\boldsymbol{E}(\boldsymbol{x}, \omega)$ to the source current density, and the integration is extended over the whole volume $V$ where

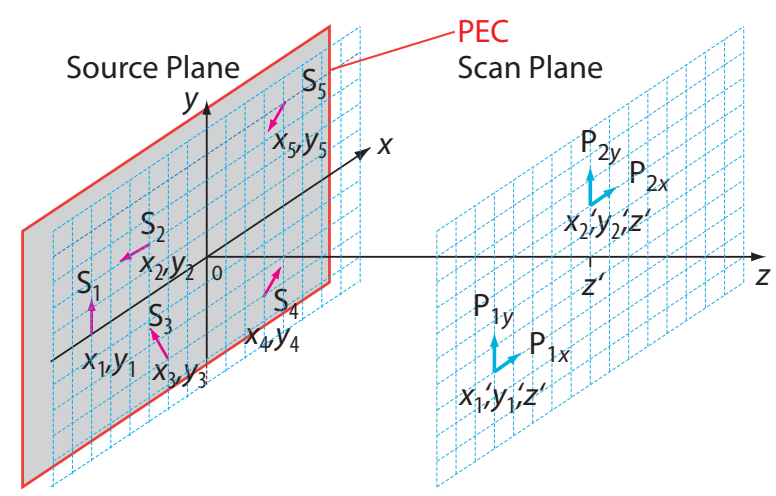

Fig. 2: EMI sources sampled at a scan plane [10].

$\boldsymbol{J}(\boldsymbol{x}, \omega)$ is non-vanishing [4], [10]. We obtain from (8) and (9) the relation cyclic correlation spectra of the excitation current density and the excited electric field as

$$
\begin{aligned}
& \underline{\Gamma}_{E, n}\left(\boldsymbol{x}_{a}, \boldsymbol{x}_{b}, \omega\right)=\iint_{V} \boldsymbol{G}_{E J}\left(\boldsymbol{x}_{a}-\boldsymbol{x}_{a}^{\prime}, \omega\right) \times \\
& \underline{\underline{\Gamma}}_{J, n}\left(\boldsymbol{x}_{a}, \boldsymbol{x}_{b}, \omega\right) \boldsymbol{G}_{E J}^{\dagger}\left(\boldsymbol{x}_{b}-\boldsymbol{x}_{b}^{\prime}, \omega-n \omega_{0}\right) d^{3} x_{a}^{\prime} d^{3} x_{b}^{\prime} .
\end{aligned}
$$

In the near-field the Green's dyadic is given by

$$
\boldsymbol{G}_{E J}(\boldsymbol{x}, \omega)=\left[g_{1}(\boldsymbol{x}, \omega) \mathbf{1}+g_{2}(\boldsymbol{x}, \omega) \boldsymbol{x} \boldsymbol{x}^{T}\right] e^{-\jmath \beta(\omega)|\boldsymbol{x}|}
$$

with

$$
\begin{aligned}
& g_{1}(\boldsymbol{x}, \beta)=-\frac{\jmath Z_{F 0} \beta^{2}}{4 \pi}\left[\frac{1}{\beta|\boldsymbol{x}|}+\frac{\jmath}{\beta^{2}|\boldsymbol{x}|^{2}}-\frac{1}{\beta^{3}|\boldsymbol{x}|^{3}}\right], \\
& g_{2}(\boldsymbol{x}, \beta)=\frac{\jmath Z_{F 0} \beta^{2}}{4 \pi}\left[\frac{1}{\beta|\boldsymbol{x}|^{3}}+\frac{3 \jmath}{\beta^{2}|\boldsymbol{x}|^{4}}-\frac{3}{\beta^{3}|\boldsymbol{x}|^{5}}\right]
\end{aligned}
$$

where $Z_{F 0}=\sqrt{\varepsilon_{0} / \mu_{0}}$ is the free space wave impedance, and the ${ }^{T}$ denotes the transpose of the vector.

\section{Correlation of the Signals Measured by two PROBES IN THE NEAR-FIELD}

Following [9], [10] we extend the method applied for the modeling of stationary electromagnetic fields to the investigation of CS electromagnetic fields. The field of localized point-like CS stochastic source currents at the locations $\boldsymbol{x}_{\mu}$ is represented by the CCS dyadic

$$
\begin{gathered}
\underline{\Gamma}_{J, n}\left(\boldsymbol{x}_{a}, \boldsymbol{x}_{b}, \omega\right)=\sum_{\mu=1}^{N} \sum_{\nu=1}^{N} l_{\mu} l_{\nu} C_{I, n, \mu \nu}(\omega) \boldsymbol{\Omega}_{\mu} \boldsymbol{\Omega}_{\nu}^{T} \times \\
\delta\left(\boldsymbol{x}_{\boldsymbol{a}}-\boldsymbol{x}_{\mu}\right) \delta\left(\boldsymbol{x}_{\boldsymbol{b}}-\boldsymbol{x}_{\nu}\right) .
\end{gathered}
$$

The cyclic correlation spectra matrix elements of the dipole currents $I_{T, \mu}(\omega)$ is given by

$$
C_{I, n, \mu \nu}(\omega)=\int_{-\infty}^{+\infty} c_{I, n, \mu \nu}(\tau) \mathrm{e}^{j n \omega_{0} t} d t
$$

where the cyclic correlation time-domain matrix elements are

$$
c_{I, n, \mu \nu}(\tau)=\frac{1}{T_{0}} \int_{-T_{0} / 2}^{T_{0} / 2}\left\langle i_{T, \mu}(t) i_{T, \nu}(t-\tau)\right\rangle \mathrm{e}^{-\jmath n \omega_{0} t} d t .
$$




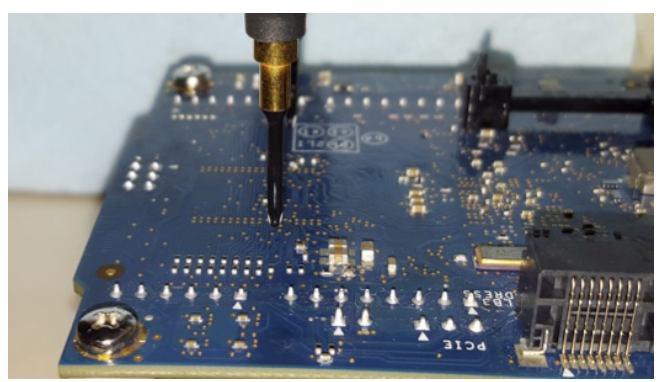

Fig. 3: Measurement setup.

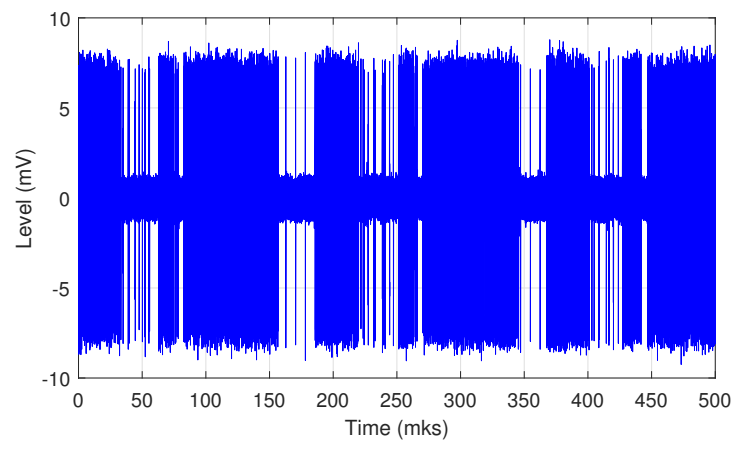

Fig. 4: Realization of the stochastic signal.

Finally, with (10) we obtain the CCS dyadic of the stochastic electric field vectors at the points $\boldsymbol{x}_{a}$ and $\boldsymbol{x}_{b}$ as

$$
\begin{gathered}
\underline{\Gamma}_{E, n}\left(\boldsymbol{x}_{a}, \boldsymbol{x}_{b}, \omega\right)=\sum_{\mu=1}^{N} \sum_{\nu=1}^{N} l_{\mu} l_{\nu} C_{I, n, \mu \nu}(\omega) \times \\
\boldsymbol{G}_{E J}\left(\boldsymbol{x}_{a}-\boldsymbol{x}_{\mu}, \omega\right) \boldsymbol{\Omega}_{\mu} \boldsymbol{\Omega}_{\nu}^{T} \boldsymbol{G}_{E J}^{\dagger}\left(\boldsymbol{x}_{b}-\boldsymbol{x}_{\nu}, \omega-n \omega_{0}\right) .
\end{gathered}
$$

\section{EXPERIMENTAL INVESTIGATION AND RESULTS}

Initial measurements are presented in this paper for the purpose of investigating the structure of the emission spectra so as to try and design efficient means of characterizing the field-field correlation spectrum of real devices by measurement. Initially, a single probe scan above a PCB is performed to establish the location of major sources of radiation. Two Langer EMV-Technik RF R50-1 magnetic field probes are connected to the input ports of an Agilent MSO8104A digital time domain oscilloscope. Figure 3 shows the two probe scanning measurement in progress for an Arduino Galileo board. Figure 4 shows a typical realization of the stochastic signal measured by a near-field probe of the tangential magnetic field radiated by the Arduino Galileo board which is programmed for data transfer between central processor unit (CPU) and internal memory.

The structure of the measured realization reveals the presence of a regularity in the measured signal and presumes cyclostationary properties of the observed stochastic process.

Implementation of the ensemble averaging procedure assumes the necessity of a synchronization with the periodic process intrinsic to the technology for data transfer in digital devices. The synchronized ensemble of the measured realiza-

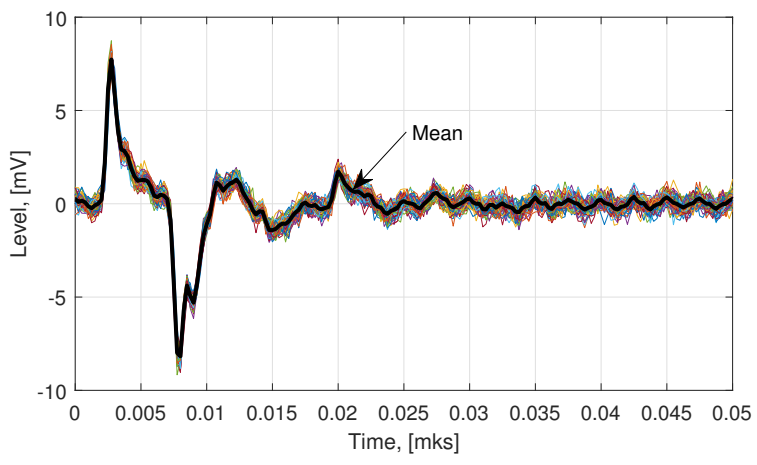

Fig. 5: Ensemble of periodic signals.

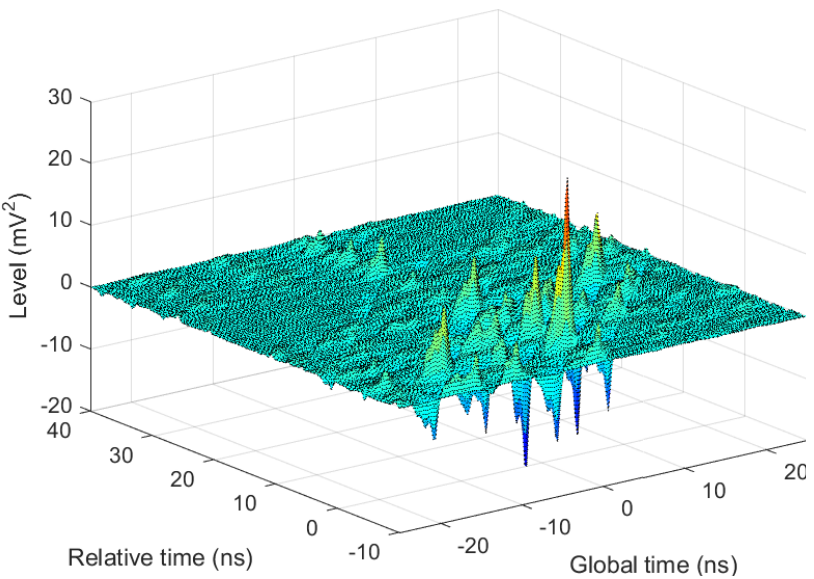

Fig. 6: Periodic Autocorrelation Function.

tions and its mean, which is a time dependent function, are shown in the Fig. 5.

The information region of the periodic autocorrelation function (4) of the cyclostationary stochastic process is presented in Fig. 6. This two-dimensional function shows the dependence of the autocorrelation from global time and relative time shift of the stochastic process. The periodic correlation function can be represented by Fourier series expansion assuming that $N$ is odd as

$$
\rho_{X}[n, m]=\sum_{l=-(N-1) / 2}^{(N-1) / 2} R_{X}^{l}[m] e^{\jmath \frac{2 \pi l n}{N}},
$$

where $R_{X}^{l}[m]$ are Fourier series coefficients

$$
R_{X}^{l}[m]=\frac{1}{N} \sum_{n=-(N-1) / 2}^{(N-1) / 2} \rho_{X}[n, m] e^{-\jmath \frac{2 \pi l n}{N}} .
$$

The Fourier coefficients $R_{X}^{l}[m]$ for the time-discrete process, compare (6), are called cyclic autocorrelation functions, where $l / N, \forall l \in \mathbb{N}$ are the cyclic frequencies. This twodimensional function, visualized in Fig. 7, shows the structure of the Fourier series spectrum for different relative time shifts of the stochastic process: the correlation interval around $40 \mathrm{~ns}$ and the spectral width of nearly $1000 \mathrm{MHz}$ of the cyclostationary stochastic process is clearly seen. The Fourier 


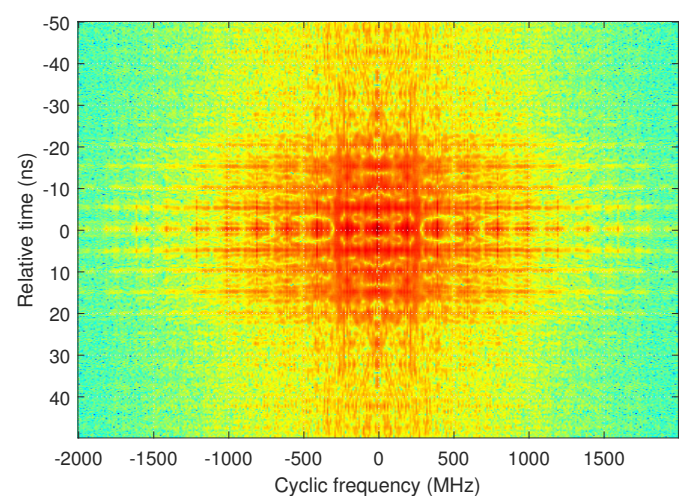

Fig. 7: Cyclic Autocorrelation Function.

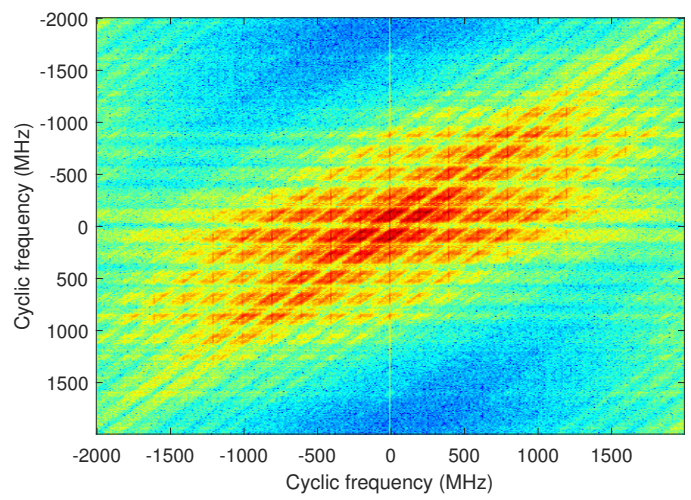

Fig. 8: Spectral Correlation Density Function.

series transform of the cyclic autocorrelation function gives the spectral correlation density function as

$$
S_{X}^{l}[v]=\frac{1}{N} \sum_{m=-(N-1) / 2}^{(N-1) / 2} R_{X}^{l}[m] e^{-\jmath \frac{2 \pi v m}{N}},
$$

where $v$ is a discrete frequency. This function, graphed in Fig. 8, represents the spectral correlation of global (horizontal axis) and relative cyclic frequencys content. In particular, spectral correlation reveals a coherent clock frequency of 400 $\mathrm{MHz}$ and its harmonics (vertical red lines) while cyclic autocorrelation function in Fig. 6 represents only cyclic periodic properties of the cyclostationary process. The experimental results demonstrate the ability of parameter estimation for the radiated emissions by using the cyclostationary stochastic characteristics obtained from the measured random signals in the near-field region of the digital device.

\section{CONCLUSION AND OUTLOOK}

We presented measurements and analysis of correlation data of CS noisy EM fields. We have extended the near-field correlation measurement methods to CS EM fields and could provide, based on these methods, parameter estimation for CS processes on the investigated Adruino microcontroller board.

\section{ACKNOWLEDGMENT}

This work was supported in part by the German Research Foundation (DFG), under grant no. Ru 314/45-1, the Russian
Foundation for Basic Research Grant 15-08-08951, by an STSM grant from COST ACTION IC1407 'ACCREDIT', and by the European Union's Horizon 2020 research and innovation programme under grant no. 664828 (NEMF21).

\section{REFERENCES}

[1] D. Thomas, C. Obiekezie, S. Greedy, A. Nothofer, and P. Sewell, "Characterisation of noisy electromagnetic fields from circuits using the correlation of equivalent sources," in 2012 Int. Symp. on Electromagnetic Compatibility (EMC EUROPE), Sep. 2012, pp. 1-5.

[2] D. Thomas, C. Obiekezie, and X. Tong, "Equivalent dipole models of electromagnetic emissions from near-field scanning," Electromagnetic Compatibility Magazine, IEEE, vol. 4, no. 3, pp. 74-78, rd 2015.

[3] A. Baev, A. Gorbunova, M. Konovalyuk, Y. Kuznetsov, and J. A. Russer, "Stochastic EMI sources localization based on ultra wide band near-field measurements," in European Microwave Conference (EuMC), Nuremberg, 6-10 Oct. 2013, pp. 1131-1134.

[4] J. A. Russer, F. Mukhtar, O. Filonik, G. Scarpa, and P. Russer, "Modelling of noisy EM field propagation using correlation information of sampled data," in IEEE Int. Conf. on Numerical Electromagnetical Modeling and Optimization NEMO2014, Pavia, Italia, May 14-16 2014.

[5] J. A. Russer, T. Asenov, and P. Russer, "Sampling of stochastic electromagnetic fields," in IEEE MTT-S Int. Microw. Symp., 2012, pp. 1-3.

[6] A. Baev, A. Gorbunova, M. Konovalyuk, Y. Kuznetsov, and J. A. Russer, "Planar stochastic sources localization algorithm in EMC problems," in Int. Conf. on Electromagnetics in Advanced Applications (ICEAA), 2013. IEEE, September 9-13 2013, pp. 440-443.

[7] C. Smartt, D. Thomas, H. Nasser, M. Baharuddin, G. Gradoni, S. Creagh, and G. Tanner, "Challenges of time domain measurement of fieldfield correlation for complex PCBs," in 2015 IEEE Int. Symp. on Electromagnetic Compatibility (EMC), Aug 2015, pp. 953-958.

[8] J. A. Russer and P. Russer, "Stochastic electromagnetic fields," in German Microwave Conference (GeMIC), Mar. 2011, pp. 1-4.

[9] J. A. Russer, G. Gradoni, G. Tanner, S. C. Creagh, D. Thomas, C. Smartt, and P. Russer, "Evolution of transverse correlation in stochastic electromagnetic fields," in IEEE MTT-S Int. Microw. Symp., 2015, pp. 1-3.

[10] J. A. Russer and P. Russer, "Modeling of noisy EM field propagation using correlation information," IEEE Transactions on Microwave Theory and Techniques, vol. 63, no. 1, pp. 76 - 89, Jan. 2015.

[11] J. A. Russer, N. Uddin, A. S. Awny, A. Thiede, and P. Russer, "Nearfield measurement of stochastic electromagnetic fields," Electromagnetic Compatibility Magazine, IEEE, vol. 4, no. 3, pp. 79-85, rd 2015.

[12] G. Gradoni, S. C. Creagh, G. Tanner, C. Smartt, and D. W. P. Thomas, "A phase-space approach for propagating field-field correlation functions," New Journal of Physics, vol. 17, no. 9, p. 093027, 2015.

[13] J. A. Russer, A. Cangellaris, and P. Russer, "Correlation transmission line matrix (CTLM) modeling of stochastic electromagnetic fields," in IEEE MTT-S Int. Microwave Symposium (IMS), May 2016, pp. 1-3.

[14] E. Serpedin, F. Panduru, I. Sar, and G. B. Giannakis, "Bibliography on cyclostationarity," Signal Processing, vol. 85, no. 12, pp. 2233-2303, Dec. 2005

[15] W. A. Gardner, A. Napolitano, and L. Paura, "Cyclostationarity: Half a century of research," Signal Processing, vol. 86, no. 4, pp. 639-697, Apr. 2006.

[16] J. Antoni, "Cyclic spectral analysis in practice," Mechanical Systems and Signal Processing, vol. 21, no. 2, pp. 597-630, Feb. 2007.

[17] —_, "Cyclostationarity by examples," Mechanical Systems and Signal Processing, vol. 23, no. 4, pp. 987-1036, May 2009.

[18] P. P. J. Schreier and P. L. L. Scharf, Statistical Signal Processing of Complex-Valued Data: The Theory of Improper and Noncircular Signals, 1st ed. Cambridge, New York: Cambridge University Press, 2010.

[19] J. A. Russer, P. Russer, M. Konovalyuky, A. Gorbunova, A. Baev, and Y. Kuznetsov, "Analysis of cyclostationary stochastic electromagnetic fields," in Int. Conf. on Electromagnetics in Advanced Applications (ICEAA), 2015. IEEE, September 7-11 2015, pp. 1452-1455.

[20] $\_$, "Near-field propagation of cyclostationary stochastic electromagnetic fields," in Int. Conf. on Electromagnetics in Advanced Applications (ICEAA), 2015. IEEE, September 7-11 2015, pp. 1456-1459.

[21] W. A. Gardner, Introduction to Random Processes, With Applications to Signals \& Systems, 2nd ed. New York: Mcgraw-Hill, 1990.

[22] S. K. Jayaweera, Signal Processing for Cognitive Radios, 1st ed. Hoboken, New Jersey: Wiley, Dec. 2014. 